\title{
Recent progress in holographic display technology
}

\section{Ling Li, Keyu Tao}

Ling Li, Keyu Tao, "Recent progress in holographic display technology," Proc. SPIE 10452, 14th Conference on Education and Training in Optics and Photonics: ETOP 2017, $104524 Z$ (16 August 2017); doi: 10.1117/12.2269490

SDIE Event: 14th Conference on Education and Training in Optics and Photonics, SPIE. ETOP 2017, 2017, Hangzhou, China 


\title{
Recent Progress in Holographic Display Technology
}

\author{
Ling Li*a, Keyu Tao ${ }^{\mathrm{b}}$ \\ ${ }^{a}$ College of Optoelectronic Engineering, Shenzhen University, Shenzhen, China; ${ }^{b}$ College of \\ Electronic Science and Technology, Shenzhen University, Shenzhen, China
}

\begin{abstract}
Optics is one of the most important courses for students whose majors are optics-related. In this article, we would like to share our teaching experiences of introducing the newest holographic display technologies which are understandable for undergraduate students. According to the feedbacks from the students, it can stimulate the students' interest in the course by introducing the up-to-date research achievements. And the students can understand the basic concepts and principles quite well while we link them with the scientific frontier. We think it is important for the course instructors to keep in steps with the discipline frontier, thus we can introduce the newest contents to the students. The teaching mode is more flexible, the teaching process will be more attractive.
\end{abstract}

Keywords: optics, quality education, holography, holographic display

\section{INTRODUCTION}

Optics is one of the oldest disciplines, which shows charming applications in our modern life. As a basic course for students in science and engineering, it is very important for them to master the basic optical concepts and principles. However, a considerable number of students reflect that the lesson is difficult to learn. They suffer from the mathematic derivations and literal expressions. How to arise students' enthusiasm for learning in order to achieve better teaching effect in optical course? We have tried to add some up-to-date achievements of the scientific frontier or related hot topics in the course, which can attract the students' interest quite well. Here we will share our teaching experiences of introducing the concepts and applications of holography.

We begin the course with two posters, one is from the 3D film named "Kung Fu Panda", and the other is from the 2015 spring festival gala evening program "Shu embroidery". Then we give out the question to the students: "Are they holographic displays?" The students are familiar with these two issues. However, no one can exactly explain the display principles. Figure 1 (a) shows the three-dimensional stereoscopic film (3D) film theory. It can enhance the illusion of depth perception. In order to watch a stereoscopic motion picture, two images are superimposed onto the screen through different polarizing filters [1]. The audience wear eyeglasses that also contain a pair of polarizing filters with different orientations. As each filter passes only the similarly polarized light and blocks the differently polarized light, each eye sees a different image. This can produce a three-dimensional effect by projecting the same scene into both eyes. Figure 1 (b) shows the stage illusions (Pepper's Ghost) principle. The images are projected to the imaging screen, and then reflected towards the

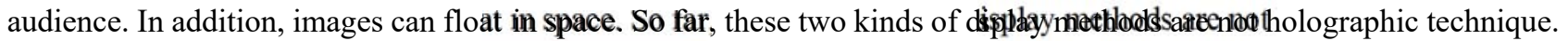

(a)

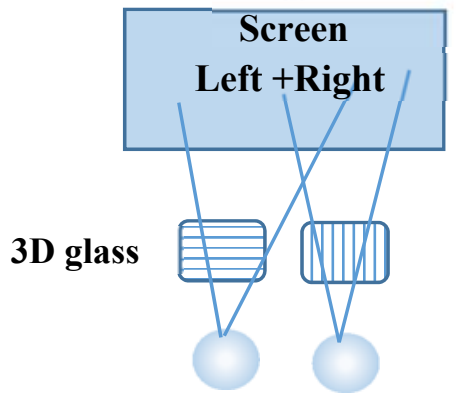

Left eye Right eye (b)

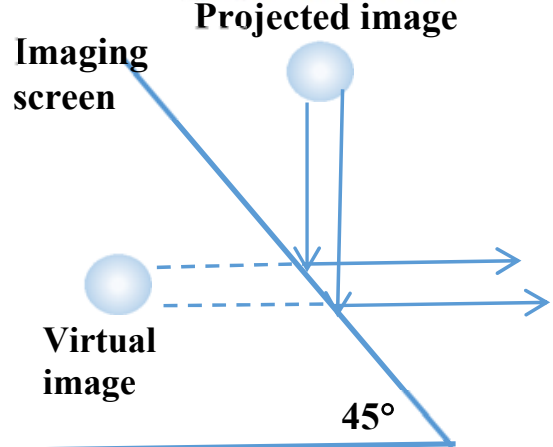

Figure 1. Illustration of 3D film and stage illusions (Pepper's Ghost).

14th Conference on Education and Training in Optics and Photonics: ETOP 2017, edited by Xu Liu,

Xi-Cheng Zhang, Proc. of SPIE Vol. 10452, 104524Z · (C) 2017 ICO, IEEE, OSA, SPIE

CCC code: $0277-786 \mathrm{X} / 17 / \$ 18 \cdot$ doi: $10.1117 / 12.2269490$ 


\section{TRADITIONAL HOLOGRAPHIC IMAGING}

The students are now wondering what a true holographic display is. We then show several holographic images to the students. Figure 2 shows a holographic image of some shells. It is a reflection hologram, and it can be viewed using a white-light illumination source. We can see the three dimensional shells in the photo clearly without glasses or other optical elements. However, the hologram is not a true image, it is the encoding of the light field as an interference pattern $[2,3]$.

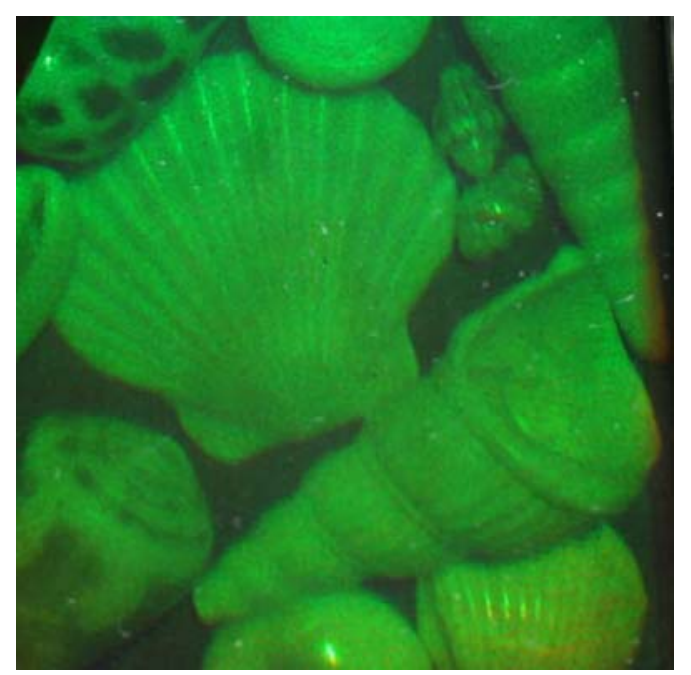

Figure 2. A holographic image of shells.

Now, the students are interested in the formation of the holographic image. A simple hologram can be taken by superimposing two coherent light beams on a holographic recording film. As shown in figure 3, laser light emits after the shutter, and is split into two beams. One is the object beam and the other is the reference beam. The object beam is expanded by passing it through a lens and used to illuminate the object. The recording film is located where the reflected or scattered object beam will strike it. The reference beam is expanded and made to shine directly on the film, where it interacts with the object beam to create the interference pattern. The recorded light pattern is a diffraction grating. When it is illuminated by only one of the beam used to create it, it can be shown that one of the diffracted beam emerges at the same angle as that at which the second beam is originally incident so that the second beam has been "reconstructed".

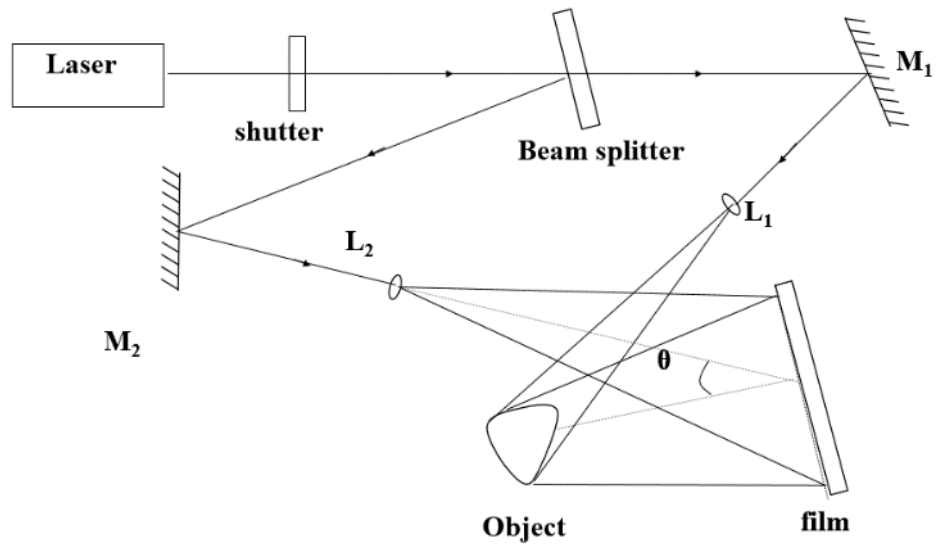

Figure 3. Illustration for recording system of hologram.

The mathematical expressions of the process are as follows:

$$
\widetilde{U}_{O}(Q)=A_{O} e^{i \varphi_{O}(Q)}, \quad \tilde{U}_{R}(Q)=A_{R} e^{i \varphi_{R}(Q)}
$$


$\widetilde{U}_{O}$ and $\widetilde{U}_{R}$ are the complex amplitudes of object beam and reference beam at arbitrary point Q on the recording film, respectively. The amplitude and phase of the lights are represented by the complex amplitude. The light intensity at point $\mathrm{Q}$ is:

$$
I(Q)=\left(\widetilde{U}_{O}+\widetilde{U}_{R}\right)\left(\widetilde{U}_{O}+\widetilde{U}_{R}\right)^{*}=A_{O}^{2}+A_{R}^{2}+\widetilde{U}_{O} \widetilde{U}_{R}^{*}+\widetilde{U}_{R} \widetilde{U}_{O}^{*}
$$

If the film is developed, its transmittance $t$ is proportional to the light intensity, and is given by:

$$
t(Q)=t_{0}+\beta I(Q)=t_{0}+\beta\left(A_{O}^{2}+A_{R}^{2}+\widetilde{U}_{O} \widetilde{U}_{R}^{*}+\widetilde{U}_{R} \widetilde{U}_{O}^{*}\right)
$$

where $\beta$ is a constant.

When the developed film is illuminated by the reference beam, the light transmitted intensity through the film $\tilde{U}_{\mathrm{T}}$ is equal to the transmittance $t$ multiplied by the reference beam amplitude. Only considering the zeroth and first order of diffraction, the transmitted intensity is:

$$
\widetilde{U}_{T}=\widetilde{U}_{R}^{\prime} t=\left(t_{0}+\beta A_{R}^{2}+\beta A_{O}^{2}\right) \widetilde{U}_{R}^{\prime}+\beta A_{R}^{\prime} A_{R}\left[e^{i\left(\varphi_{R}^{\prime}-\varphi_{R}\right)} \widetilde{U}_{O}+e^{i\left(\varphi_{R}^{\prime}+\varphi_{R}\right)} \widetilde{U}_{O}^{*}\right]
$$

where $\widetilde{U}_{R}^{\prime}=A_{R}^{\prime} e^{i \varphi_{R}^{\prime}}$.

\section{THE LATEST HOLOGRAPHIC DISPLAY TECHNIQUE}

Based on holographic display technique, viewers can see 3D images without the aid of any special glasses. The following are some types of latest holographic display techniques.

Surface plasmon holographic display [4-8]: Miyu Ozaki et al use surface plasmon polariton (SPP) [4-7] to form 3D color holography with white-light illumination [8]. They record the hologram on a photoresist as an interference pattern between a light field coming from the object as scattered light and the unscattered reference beam. Exposure is repeated three times with rotation of the illumination angle for a single color hologram recording. A thin metal film is then coated on the photoresist hologram. In the image reconstruction process, the SPP is excited by a component of white light that is incident on the metal film through a prism with an angle satisfying the condition of total internal reflection (figure 4a). The SPP associates with a nonradiative evanescent light wave on metal film and then is converted by the grating component of hologram into a radiative light field, which represents the reconstructed wavefront of light that scattered at the object. The reconstruction of the prerecorded object is seen with the eyes through an SPP hologram in color. The reference beam or the zeroth-order diffraction as background beam does not exist in reconstruction for this configuration because the illumination of the hologram is made by total internal reflection. Individual colors are reconstructed by illumination at corresponding incident angles by satisfying the above relationship for each color. Figure $4 \mathrm{~b}$ shows the optical setup for reconstructing a three-color object in different azimuthal and incident angle sets. Figure $4 \mathrm{c}$ shows the reconstruction of an apple with a leaf from the thin film plasmon hologram.
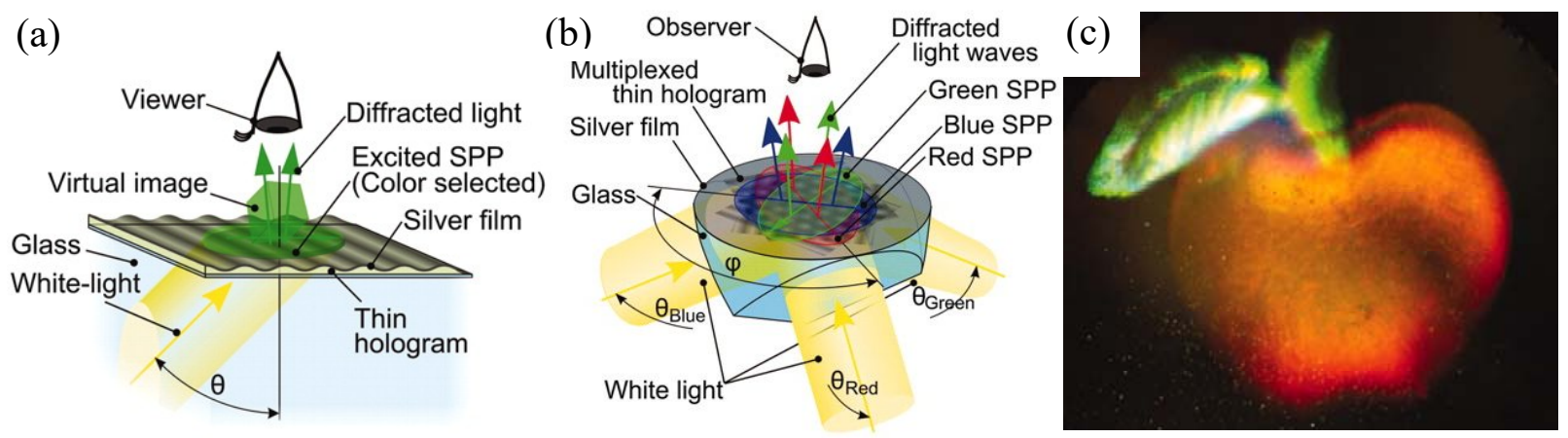

Figure 4. Hologram with surface Plasmon [8]. 
Touchable display with laser induced plasma [9-11]: A high-intensity laser can excite a physical matter to emit light at an arbitrary 3D position $[9,10]$. Yoichi Ochiai et al report a method of rendering aerial and volumetric graphics using femtosecond lasers [11]. There are two methods of rendering graphics with a femtosecond laser in air: Producing holograms using spatial light modulation technology, and scanning of a laser beam by a galvano mirror. They use femtosecond lasers with pulse durations of 30-100 fs, and $269 \mathrm{fs}$. This leads to safer plasma generation than nanosecond lasers. In addition, they use an optical device, called the spatial light modulator (SLM), to modify the phase of light rays and produce various spatial distributions of light based on interference. The production of an in-air SLM-based laserplasma graphics that enables physical contact and interaction by ultra-short pulse duration laser is shown in figure 5 .
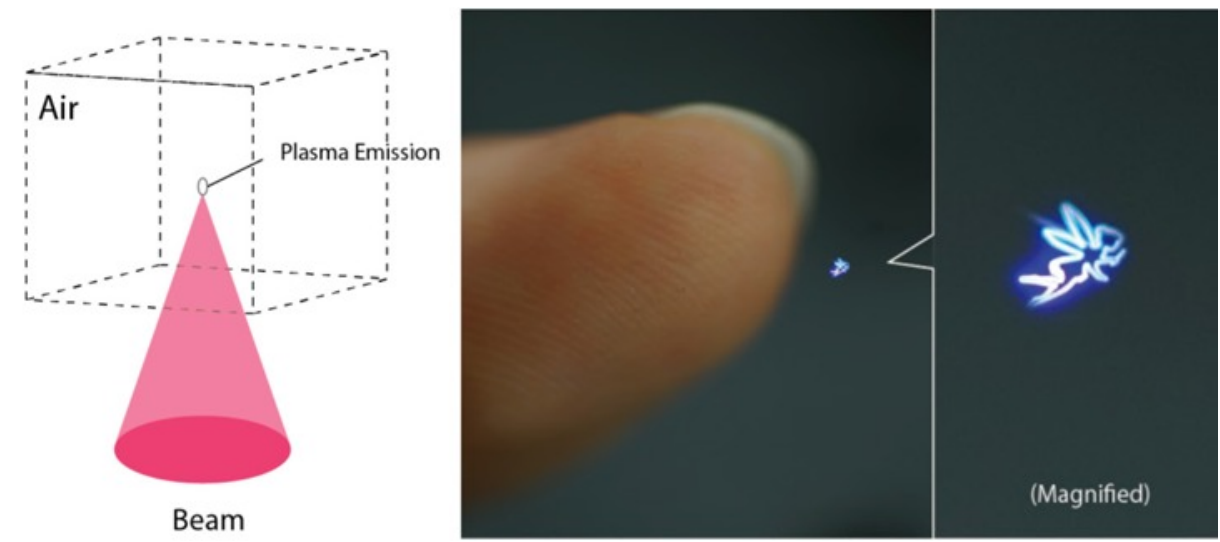

Figure 5. Touchable display with laser induced plasma [11].

\section{CONCLUSION}

According to the feedbacks from the students, it can stimulate the students' interest in the course by introducing the up-todate research achievements. And the students can understand the basic concepts and principles quite well while we link them with the scientific frontier. We think it is important for the course instructors to keep in steps with the discipline frontier, thus we can introduce the newest contents to the students. The teaching mode is more flexible, the teaching process will be more attractive.

\section{REFERENCES}

[1] Zone, R., [Stereoscopic cinema \& the origins of 3D film], University Press of Kentucky, Kentucky, (2007).

[2] Born, M., and Wolf, E., [Principles of Optics], Combridge University Press, Combridge, (1999).

[3] Zhao, K. and Zhong, X., [Optics], Peking University Press, Beijing, (2009).

[4] Raether, H., [Surface Plasmons on Smooth and Rough surfaces and on Gratings], Springer, Berlin, (1988).

[5] Heber, J., "Surfing the wave", Nature, 461, 720-722 (2009).

[6] Cowan, J. J., "The surface plasmon resonance effect in holography", Opt. Commun., 5, 69-72 (1972).

[7] Wang, G. P., Sugiura, T., and Kawata, S., "Holography with surface-plasmon-coupled waveguide modes", Appl. Opt., 40, 3649-3653 (2001).

[8] Ozaki, M., Kato, J., Kawata, S., "Surface-Plasmon Holography with White-Light Illumination", Science, 332(6026): 218-220 (2011).

[9] Keldysh, L. V., "Ionization in the field of a strong electromagnetic wave", Soviet Physics JETP 20, 1307-1314, (1965).

[10] Hayasaki, Y., Sugimoto, T., Takita, A., and Nishida, N., "Variable holographic femtosecond laser processing by use of a spatial light modulator", Appl. Phys. Lett., 87, 031101, (2005).

[11] Ochiai, Y., Kumagai, K., Hoshi, T., Rekimoto, J., Hasegawa, S., Hayasak, Y., "Fairy lights in femtoseconds: aerial and volumetric graphics rendered by focused femtosecond laser combined with computational holographic fields", Acm Transactions on Graphics, 35(2), 17:1-17:14 (2016). 